\title{
Maternal Folate Concentration in Early Pregnancy and Newborn Telomere Length
}

\author{
Sonja Entringer ${ }^{a, j} \quad$ Elissa S. Epel ${ }^{\text {h }}$ Jue Lin ${ }^{i}$ Elizabeth H. Blackburn ${ }^{i} \quad$ Claudia Buss $^{a, j}$ \\ Babak Shahbaba $^{d}$ Daniel L. Gillen ${ }^{d}$ Raman Venkataramanan ${ }^{f}$ Hyagriv N. Simhan ${ }^{9}$
}

Pathik D. Wadhwa a-c, e

Departments of a Pediatrics, ${ }^{\mathrm{b}}$ Obstetrics and Gynecology, ${ }^{\mathrm{c} E p i d e m i o l o g y},{ }^{\mathrm{d}}$ Statistics, and e Psychiatry and Human Behavior, University of California, Irvine, Calif.; Department of ${ }^{f}$ Pharmaceutical Sciences, and ${ }^{g}$ Obstetrics, Gynecology and Reproductive Sciences, University of Pittsburgh, Pittsburgh, Pa., Departments of h Psychiatry and 'Biochemistry and Biophysics, University of California, San Francisco, Calif., USA; 'j Department of Medical Psychology, Charité Universitätsmedizin Berlin, Germany

\section{Key Words}

Folate · Pregnancy · Telomere biology · Fetal programming

\begin{abstract}
Background/Aims: Telomere biology plays a fundamental role in genomic integrity and cell physiology. The newborn setting of telomere length (TL) likely has important implications for telomere dynamics over the lifespan; however, its determinants are poorly understood. Folate is essential for DNA integrity. The maternal compartment is the only source of folate for the developing fetus. We, therefore, tested the hypothesis that variation in maternal folate during pregnancy is associated with newborn TL. Methods: A prospective, longitudinal study was conducted in 119 mother-newborn dyads. Eligible mothers were enrolled at $9.5(S D \pm 2.1)$ weeks gestation and followed through birth. Concentrations of maternal serum folate were measured in the first trimester of pregnancy. Newborn telomere length was measured in cord blood mononuclear cells (CBMC). Results: After accounting for the effects of other established determinants of newborn $\mathrm{TL}$, each $10 \mathrm{ng} / \mathrm{ml}$ increase in maternal total folate was associated with a $5.8 \%$ increase in median TL $(p=0.03)$. The median TL in newborns of mother in the lowest quartile of total
\end{abstract}

\section{KARGER 125}

(C) 2015 S. Karger AG, Base

0250-6807/15/0664-0202\$39.50/0

E-Mail karger@karger.com

www.karger.com/anm folate levels was approximately $10 \%$ shorter than that of newborns of mothers in the highest folate quartile. Conclusions: Our findings suggest that fetal TL exhibits developmental plasticity, and provide evidence that maternal nutrition may exert a 'programming' effect on this system.

(c) 2015 S. Karger AG, Basel

\section{Introduction}

Telomere biology has emerged in recent years as playing a fundamental role in genomic integrity, cellular regeneration, physiology, aging, disease risk and mortality [1]. Telomere biology refers to the structure and function of telomeres, tracts of non-coding tandem repeats of simple DNA sequences $\left.\left(5^{\prime} \text {-(TTAGGG) }\right)_{n}-3^{\prime}\right)$ and their bound proteins that cap the ends of linear chromosomes, and telomerase, the cellular reverse transcriptase enzyme that adds telomeric DNA to telomeres. Without replenishment by telomerase and other activities, telomeres lose

Pathik D. Wadhwa, MD, PhD

UC Irvine Development, Health and Disease Research Program University of California, Irvine, School of Medicine

3117 Gillespie Neuroscience Research Facility (GNRF), Irvine, CA 92697 (USA)

E-Mail pwadhwa@uci.edu

Hyagriv N. Simhan, MD, MS

Department of Obstetrics, Gynecology and Reproductive Sciences

University of Pittsburgh

300 Halket Street, Pittsburgh, PA 15213 (USA)

E-Mail hsimhan@mail.magee.edu 
approximately $50-150$ base pairs (bp) with each cell division, and their decline to critically short lengths leads to loss of telomere function. This, in turn, can cause the activation of DNA damage checkpoint responses, impaired stem and other cell functions, chromosomal fusions and genome instability [1]. The loss of the integrity of telomeres not only affects the replicative capacity of the cell but also enforces a self-perpetuating pathway of global epigenetic changes that alter overall chromatin and transcriptional properties, ultimately leading to cell senescence and aging. Telomere shortness has been associated in numerous studies across diverse populations with the occurrence and progression of common chronic disorders such as cardiovascular disease, hypertension, atherosclerosis, heart failure, type 2 diabetes [2], and with earlier mortality [3].

The initial, or newborn, setting of telomere length (TL) represents a critically important characteristic of an individual's telomere biology system [4]. It constitutes one of two major determinants of TL at any subsequent age (the other determinant is TL attrition over time) [1]. A reduction in the newborn TL could therefore confer greater susceptibility in later life for pathophysiological outcomes, highlighting the importance of understanding factors that determine an individual's newborn TL [4-6]. Indeed, findings from animal models suggest that TL and telomere attrition rates in early life are (i) far better predictors of realized life span than TL and attrition rates in later life, and (ii) their effects persist over and above those for risk exposures in later life [6-8]. Moreover, although TL is known to differ across tissue types, a recent study established that the rate of age-dependent TL shortening in humans appears to be similar across different somatic tissues (leukocyte, skeletal muscle, skin and fat), suggesting the observed TL differences between tissues are established in early life, and highlighting the importance of a better understanding of the determinants of inter-individual variation in the initial setting of TL at birth [9].

The determinants of newborn TL are poorly understood. Despite the relatively high heritability estimates, genetic variation (from candidate gene as well as GWAS approaches) accounts for only a small proportion of the variance in TL throughout the lifespan (e.g. $[4,10])$. This highlights the particular importance of a better understanding of intrauterine environmental factors that may contribute to newborn TL. Animal as well as human studies suggest that adverse or suboptimal conditions in intrauterine life, such as fetal growth restriction or preeclampsia, are associated with shorter offspring TL (summarized in [5]), thereby supporting the concept that the

Maternal Folate and Newborn Telomere Length setting of TL may, in part, be programmed in utero. In this context, we suggest that maternal nutritional state, and particularly folate, represents an attractive candidate variable of interest. Folate represents a nutritional measure of particular interest because it plays crucial roles in maintenance of DNA integrity and DNA methylation, both of which influence TL $[11,12]$. Studies in adults have reported significant cross-sectional associations between plasma concentrations of folate and TL $[13,14]$. The importance of folate in fetal development has previously been established in the context of DNA synthesis, cell proliferation and neural tube development [15]. The fetus is completely dependent on maternal supply for folate, which occurs via a process of unidirectional maternal-tofetal folate transport [15]. Consequently, there is a very high degree of correlation between maternal and fetal concentrations of folate [16]. Moreover, maternal folate intake during pregnancy has been associated with methylation status and related offspring phenotype [17].

Thus, given (1) the possibility that the newborn setting of TL is determined, in part, by nutrition-related intrauterine processes, (2) the established role of folate in DNA synthesis and TL maintenance, and (3) the dependence of the fetus on maternal folate, the goal of this study was to test the hypothesis that lower maternal folate concentration in pregnancy is associated with shorter telomeres in the newborn. We further hypothesized that this effect persists even after accounting for the effects of other potential determinants of newborn TL. We elected to examine the continuum of the physiologic distribution of folate, as opposed to the overt folate-deficient state, in order to achieve a more conservative test of our hypothesis and to ensure the generalizability of results to the North-American population of pregnant women (who are supplemented and consume folic acid fortified foods and hence unlikely to be folate deficient). Last, we focused on maternal folate concentration in early gestation because this is the period of formation and maturation of the original pool of hematopoietic stem cells that ensure the lifelong production of blood cells through self-renewal and differentiation into all blood lineages including peripheral leukocytes [18].

\section{Materials and Methods}

Study Participants and Procedures

The study sample was a sub-sample of women from a larger cohort of women attending prenatal care at a university-based clinic in Pittsburgh, PA and participating in a prospective, longitudinal study from early gestation through birth. This sub-sample comprised $n=119$ subjects in whom measures of maternal folate 
Table 1. Maternal and newborn characteristics of the current study sample and of the larger birth cohort from which the present sample was drawn

\begin{tabular}{|c|c|c|c|}
\hline Maternal characteristics & $\begin{array}{l}\text { Current study } \\
\text { sample }(n=119)\end{array}$ & $\begin{array}{l}\text { Larger birth } \\
\text { cohort }(n=470)\end{array}$ & $\begin{array}{l}\text { Significance of } \\
\text { group comparison* }\end{array}$ \\
\hline \multicolumn{4}{|l|}{ Sociodemographic } \\
\hline Age $^{a}$, years & $24.5 \pm 4.5$ & $24.9 \pm 5.1$ & n.s. $(p=0.46)$ \\
\hline Race/ethnicity, \% & & & n.s. $(\mathrm{p}=0.58)$ \\
\hline Non-hispanic White & $49(41)$ & $191(42)$ & \\
\hline Non-hispanic Black & $65(55)$ & $246(54)$ & \\
\hline Hispanic White & $2(1.5)$ & $16(3.4)$ & \\
\hline Hispanic Black & $3(2.5)$ & $17(3.6)$ & \\
\hline \multicolumn{4}{|l|}{ Annual family income, $\%$} \\
\hline$<$ USD10,000 & $54(45)$ & $193(41)$ & n.s. $(p=0.78)$ \\
\hline USD10,000-25,000 & $37(31)$ & $165(35)$ & \\
\hline$>\mathrm{USD} 25,000$ & $28(24)$ & $113(24)$ & \\
\hline \multicolumn{4}{|l|}{ Pregnancy } \\
\hline Pre-pregnancy body mass index ${ }^{\mathrm{a}}, \mathrm{kg} / \mathrm{m}^{2}$ & $27.5 \pm 7.3$ & $27.9 \pm 6.9$ & n.s. $(p=0.35)$ \\
\hline \multicolumn{4}{|l|}{ Parity, \% } \\
\hline 0 & $11(9)$ & $72(15)$ & n.s. $(p=0.25)$ \\
\hline 1 & $33(28)$ & $113(24)$ & \\
\hline$\geq 2$ & $75(63)$ & $285(61)$ & \\
\hline Presence of obstetric risk condition, \% & $16(13)$ & $66(14)$ & n.s. $(p=0.91)$ \\
\hline \multicolumn{4}{|l|}{ Newborn characteristics } \\
\hline Sex, females, \% & $53(45)$ & $211(45)$ & n.s. $(p=0.89)$ \\
\hline Gestational age at birth ${ }^{\mathrm{a}}$, weeks & $38.9 \pm 1.8$ & $38.8 \pm 2.2$ & n.s. $(\mathrm{p}=0.85)$ \\
\hline Birth weight ${ }^{\mathrm{a}}, \mathrm{g}$ & $3,206 \pm 521$ & $3,265 \pm 681$ & $\mathrm{p}=0.88$ \\
\hline
\end{tabular}

n.s. $=$ Not significant. ${ }^{\text {a }}$ Mean \pm SD. ${ }^{*}$ Groups were compared using t-tests or $\chi^{2}$-tests where appropriate.

and newborn cord blood DNA were available. The sociodemographic, pregnancy and newborn birth outcome characteristics of the current study population are presented in table 1. As depicted in table 1, the study population was representative of the larger cohort - there were no significant differences in maternal or newborn characteristics, obstetric risk conditions or adverse birth outcomes between mother-infant pairs included in the current report compared to those in the larger study population.

The mean gestational age at enrollment was 9.5 ( $\mathrm{SD} \pm 2.1)$ weeks. Eligible women had singleton intrauterine pregnancies and no known major pre-pregnancy medical conditions or fetal anomalies. The study was approved by the Institutional Review Board, and all mothers provided written informed consent.

At enrollment, participants completed a structured interview to collect sociodemographic characteristics, medical and reproductive history, and provided a blood sample. We ascertained race/ethnicity using the Office of Management and Budget 1997 Revisions to the Standards for the Classification of Federal Data on Race and Ethnicity (Revision of Statistical Policy Directive No. 15, Race and Ethnic Standards for Federal Statistics and Administrative Reporting). Study subjects were asked to self-report or selfidentify their race and ethnicity using two separate questions. Data on ethnicity was collected first. Respondents were offered the option of selecting one or more racial designations.

At delivery, cord blood samples were collected in a sterile manner by umbilical funipuncture. Paired maternal and cord blood samples (for folate measures and newborn TL, respectively) were available in 119 dyads. The characteristics of the study population are provided in table 1 .

\section{Quantification of Maternal Folate Levels}

Concentrations of maternal folate were measured in the first trimester of pregnancy in serum (because of superior assay precision of serum versus red cell folate [19]). All samples were processed within $20 \mathrm{~min}$ of collection. Serum was separated, aliquoted, and frozen at $-80^{\circ} \mathrm{C}$ until assay. No freeze-thaws occurred prior to assay of any samples. Total folate was defined as the sum of the 3 primary folate species, 5 -methyltetrahydrofolate (5MeTHF), 5 -formyltetrahydrofolate (5Fo-THF), and folic acid concentrations. Folate species were quantified using high-pressure liquid chromatography-tandem mass spectrometry (LC-MS/MS) on the basis of established and published methods [20] (see Supplemental Content). The total folate assay coefficient of variation (CV) was $6.9 \%$.

All women who participated in the study were supplemented with folate. The maternal blood sample was collected in a nonfasting state. Although consumption of folate-rich foods may produce a peak in blood folate after 1.5 to $2 \mathrm{~h}$, the time of day of blood sampling was $4 \mathrm{~h}$ after breakfast in the majority of subjects (94\%), and only a small number of women $(8 \%)$ had detectable concentrations of free folic acid, likely because it is present in circulation only for a very short period before it is taken up by cells [21]. These ob- 
servations support the premise that our folate measures reflect typical serum status, rather than those influenced by an immediately proximate meal.

\section{Cord Blood Mononuclear Cell TL}

We used a measure of TL in cord blood mononuclear cells (CBMCs) to characterize the newborn telomere biology system. Blood represents the most commonly used measure of telomere biology in human epidemiological studies because of its ready availability and the importance of the integrity of the immune system for health and disease. Although adult TL varies by cell and tissue type, an important feature of newborn TL is that it is tightly synchronized across various tissues [22] and among different hematopoietic cells in cord blood [23], thereby providing additional justification for the selection of this measure in the current study. The rate of age-dependent TL shortening is similar across different human somatic tissues [9], and this further supports our use of blood mononuclear cells.

Whole DNA was isolated from CBMC samples and shipped to the Blackburn laboratory at the University of California, San Francisco, where TL assays were performed. Measurement of relative TLs (T/S ratios) were performed by quantitative PCR as previously described [24]. The qPCR TL inter-assay coefficient of variation $(\mathrm{CV})$ in the current study was $8.1 \%$. The conversion from $\mathrm{T} / \mathrm{S}$ ratio to base pairs was calculated based on the mean telomeric restriction fragment (TRF) length from Southern blot analysis and the slope of the plot of mean TRF length versus T/S for these samples. This was expressed as the following formula: base pairs = $3,274+2,413^{*}(\mathrm{~T} / \mathrm{S})$. This qPCR method to assess leukocyte TL has recently been validated against Southern blot analysis [25].

Sociodemographic Characteristics, Obstetric Risk, Gestational Age at Birth and Birth Weight

Sociodemographic characteristics (maternal age, race/ethnicity, parity, income) were obtained using a structured interview at the first study visit. Pre-pregnancy BMI was abstracted from the medical record. Obstetric risk was defined as the presence of one or more of the following major medical complications in the index pregnancy: gestational diabetes, vaginal bleeding, placenta abruption, gestational hypertension, preeclampsia, or infection. Risk conditions were ascertained by medical chart review and coded as a binary variable (presence or absence of obstetric risk). Gestational age was determined by best obstetric estimate with a combination of last menstrual period and 1st or 2nd trimester ultrasound in all subjects. If last menstrual period was unknown, all subjects underwent first trimester ultrasound and gestational age was determined by first trimester crown-rump length. If last menstrual period was known, then gestational age was determined by this period, as long as gestational age was confirmed by first trimester ultrasound ( \pm 7 days) or second trimester ultrasound ( \pm 14 days). If the earliest ultrasound differed in gestational age estimate beyond the above noted confidence limits, then gestational age was established by the earliest ultrasound. Birth weight was abstracted from the newborn delivery record.

\section{Statistical Analyses}

Multiple linear regression was used to quantify the association between maternal folate concentrations and newborn TL with adjustment for the effects of other potential determinants. Adjustment covariates were specified a priori based on a review of the
Table 2. Maternal serum levels of total folate, 5-methyltetrahydrofolate (5MeTHF), and 5-formyltetrahydrofolate (5Fo-THF)

\begin{tabular}{llc}
\hline Maternal serum levels & Mean \pm SD & Range, ng/ml \\
\hline Total folate & $29.5 \pm 10.9$ & $10.0-60.0$ \\
5 MeTHF & $17.2 \pm 7.3$ & $5.0-41.0$ \\
5 Fo-THF & $10.5 \pm 6.9$ & $1.8-37.2$ \\
\hline
\end{tabular}

$\mathrm{SD}=$ Standard deviation.

published literature on the determinants of newborn telomere biology or based on their association with child or adult TL [5]. These included maternal socioeconomic status (annual family income), race/ethnicity, maternal pre-pregnancy BMI, presence of obstetric complications, maternal age, infant sex, gestational age at birth, and birth weight. Due to the skewed distribution of TL, the outcome was log-transformed to an approximately symmetric distribution. As such, the exponentiated regression parameters from the resulting linear regression model are interpretable as a relative change in median TL that is associated with a one-unit increase in corresponding model covariate. In addition, the relative difference in median TL among subjects in the upper and lower quartiles of maternal folate levels was also estimated and reported. Separate regression models were conducted for maternal total folate, 5Fo-THF and 5MeTHF levels. For all models, regression diagnostics were performed to assess the validity of standard linear regression assumptions and diagnose potential influential points. No extreme departures from general assumptions were observed and no observations were removed from the analysis. All statistical analyses were performed using SPSS version 18 , and the statistical significance level was set at $\alpha=0.05$.

\section{Results}

The mean CBMC TL, expressed as telomere repeat copy number to single gene copy number (T/S) ratio, was $2.45 \pm 0.62$ (mean $\pm \mathrm{SD}$ ), equivalent to $9,176 \pm 1,506$ base pairs (bp). This is comparable to previous reports in newborns [22]. Concentrations of maternal total folate, 5 Fo-THF and 5MeTHF, provided in table 2, indicate our study population was not folate-deficient, with variation in the normal physiological range.

After accounting for the effects of SES, race/ethnicity, maternal pre-pregnancy BMI, maternal age, obstetric complications, infant sex, length of gestation and birth weight, there was a significant, independent effect of maternal total folate on newborn TL (see table 3; fig. 1a). Specifically, a $10 \mathrm{ng} / \mathrm{ml}$ increase in total folate was associated with a $5.8 \%$ increase in median TL (95\% CI: $0.5-$ $11.3 \% ; \mathrm{p}=0.03$ ). For newborns of mothers who fall in the lowest quartile of total folate levels during pregnancy, the 
Table 3. Model estimates from regressing log-transformed newborn cord blood mononuclear cell (CBMC) telomere length ( $\mathrm{T} / \mathrm{S}$ ratio) on total folate and a priori specified adjustment variables

\begin{tabular}{lll}
\hline & $\begin{array}{l}\text { Estimated relative difference } \\
\text { in median TL (95\% CI) }\end{array}$ & $\begin{array}{l}\text { Significance, } \\
\text { p value }\end{array}$ \\
\hline Total folate, per 10 ng/ml & $1.058(1.005,1.113)$ & 0.033 \\
Annual family income, per USD1k & $0.970(0.910,1.034)$ & 0.350 \\
Race/ethnicity, Caucasian vs. African-American & $1.005(0.963,1.050)$ & 0.812 \\
Maternal pre-pregnancy BMI, per 1 kg/m² & $1.001(0.993,1.009)$ & 0.752 \\
Gestational age at birth, per week & $1.005(0.963,1.050)$ & 0.812 \\
Birth weight, per 500 g & $1.001(0.936,1.071)$ & 0.970 \\
Infant sex, female vs. male & $1.49(1.031,1.282)$ & 0.014 \\
Presence of antepartum obstetric complications & $0.792(0.677,0.926)$ & 0.005 \\
Maternal age, years & $0.996(0.983,1.009)$ & 0.539 \\
\hline
\end{tabular}

Coefficient estimates (and corresponding 95\% confidence intervals) have been exponentiated and are interpretable as the relative change in median TL (T/S ratio).

Fig. 1. a Scatterplot depicting the association between maternal total folate concentrations and residualized cord blood mononuclear cell (CBMC) telomere length (T/S ratio). Newborn CBMC telomere length was residualized (adjusted) by regressing it on the covariates (maternal SES, race/ethnicity, pre-pregnancy BMI, length of gestation, birth weight, infant sex, obstetric complications), and it is depicted in its original scale (as opposed to the log scale). b Mean adjusted cord blood PMBC telomere length (T/S ratio, \pm standard error of the mean (SEM)) for newborns of mothers who fall in the lowest quartile (low maternal total folate concentration) vs. newborns of mothers in the highest folate quartile (high maternal total folate concentration).

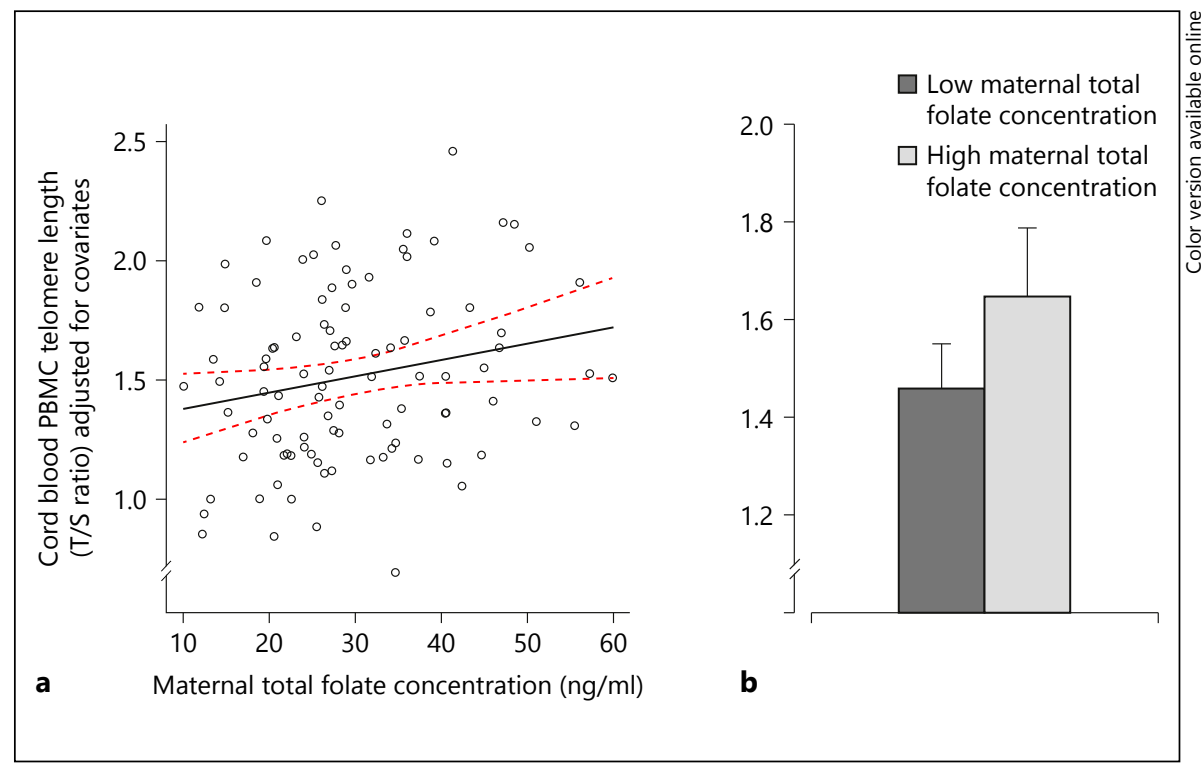

median TL was estimated to be approximately $10 \%$ shorter (equivalent to about $1,000 \mathrm{bp}$ ) than the newborns of mothers in the highest folate quartile (95\% CI: 1.5-14.3\%; see fig. 1b).

Maternal concentrations of 5MeTHF were more strongly related to newborn TL (a $10 \mathrm{ng} / \mathrm{ml}$ increase in $5 \mathrm{MeTHF}$ was associated with a $7.7 \%$ increase in median TL, 95\% CI: $0.3-15 \%$; $\mathrm{p}=0.011$ ) than total folate levels; there was no significant association between maternal levels of 5Fo-THF and newborn TL $(\mathrm{p}=0.327)$. These analyses suggest the effect of total folate on newborn TL is mainly driven by $5 \mathrm{MeTHF}$.

\section{Discussion}

This study represents, to the best of our knowledge, the first report in humans linking a maternal nutrient in pregnancy with subsequent TL in the newborn offspring. This effect persists even after adjusting for a number of other potential determinants of newborn TL. The magnitude of the effect translates to an approximately $10 \%$ difference of TL in infants of mothers in the top vs. bottom quartile of folate concentration. The variability of newborn cord blood TL is high, and although the clinical significance of this effect remains to be determined in future 
longitudinal studies, we note that based on extrapolations of available data, the magnitude of this effect appears to be approximately equivalent to that of the effect of smoking, obesity, diabetes or hypertension on adult TL [26, 27]. The concentration and variation in maternal folate in the study population are comparable to those of the current US population, indicating it is folate-sufficient by conventional thresholds. Thus, given the observed association in this folate-supplemented study population, it is possible that the effect may be even larger in newborns of mothers from folate-deficient pregnancies, such as those in the developing world.

This study challenges the conventional concept that processes producing variation in telomere maintenance manifest only during the latter part of the life span. Our study suggests that the underpinnings of these processes may start as early in life as during the intrauterine period of development. The concept of the fetal, or developmental, origins of health and disease risk posits it is the nature of conditions during intrauterine life, in interaction with genetic makeup that determines phenotypic specificity and influences subsequent health and susceptibility for complex common disorders [5]. In this context, we have proposed that impaired telomere maintenance may represent a novel pathway linking suboptimal conditions during embryonic and fetal life with subsequent child and adult health outcomes, increased susceptibility for the complex common diseases that confer the major burden of global disease, and reduced life span and longevity [5].

Given the importance of the newborn TL, a consequence of lower maternal folate in pregnancy may be the establishment of a long-term trajectory that confers increased susceptibility for many complex, common diseases via its influence on the developing telomere biology system. There is strong biological plausibility for a causal link between maternal folate concentration and offspring TL. First, evidence from animal and human studies linking other adverse conditions during fetal development with newborn TL provides support for the notion that TL may be 'programmed' in utero [5]. Second, maternal supply of folate is critically important during the fetal development for DNA synthesis and cell proliferation [15]. Third, in adults, folate concentrations have been linked with TL and maintenance of telomere integrity $[11,28]$. Thus, folate deficiency may induce TL attrition and dysfunction. Damage of DNA integrity by excessive incorporation of uracil instead of thymine in the telomeric hexameter repeat may induce breaks in the telomere sequence. Also, by virtue of its role as a methyl donor, folate may play a critical role in the maintenance of methylation

Maternal Folate and Newborn Telomere Length of cytosine, which determines the structural stability of important regions of the chromosome such as subtelomeric DNA $[11,28]$.

In addition to the effect of total folate, our study examined the separate effects of the two main folate species $5 \mathrm{MeTHF}$ and 5Fo-THF. 5Fo-THF is used for purine and pyrimidine synthesis, key precursors for DNA synthesis [28]. 5MeTHF provides methyl groups for the methylation of homocysteine (Hcy) to methionine, the precursor of S-adenosylmethionine (SAM), which is the universal methyl donor for biological methylation reactions including those of DNA and histones [28]. We note that a more complete understanding of this biological system and its contributions to both DNA synthesis and methylation requires the assessment of metabolic parameters more proximate to methylation, particularly vitamin B12 (because it is a necessary cofactor along with the provision of methyl groups from $5 \mathrm{MeTHF}$ ). Nevertheless, our findings raise the possibility that folate influences on DNA synthesis and methylation may be important in the determination of newborn TL.

We note the study was unable to assess other physiological processes during pregnancy that could potentially interact with maternal folate levels to influence the setting of newborn TL, such as maternal-placental-fetal endocrine, immune, and oxidative stress-related parameters, and also was unable to assess newborn telomerase activity. It has recently been shown that paternal age at conception correlates positively with the offspring telomere length $[29,30]$. Measures of paternal age were not available in this cohort; however, all analyses were adjusted for maternal age, and maternal and paternal ages at conception were highly correlated [31]. Another limitation of the current study was that fasting maternal blood samples were not available for assessment of maternal folate levels. Future studies are warranted that incorporate these features and also conduct longitudinal follow-up of telomere dynamics from birth into infancy, childhood and beyond, along with the concurrent characterization of other infant and child phenotypes of interest.

To conclude, maternal total folate concentration in early pregnancy was significantly and positively associated with newborn cord blood TL. Questions remain regarding the molecular mechanism(s) underlying this effect and potential interactions with other biological processes. However, in so far as the newborn setting of TL is an important determinant of subsequent telomere biology-related processes and health outcomes, the current finding represents an important step because, first, it adds 
evidence to the growing awareness that age-related complex, common disorders may have their foundations very early in life, and second, it points to a potentially modifiable factor for possible clinical intervention with implications for primary prevention.

\section{Funding Sources}

This study was supported by US PHS (NIH) grants RO1 HD06028, PO1 HD-047609, HD-065825, R01 HD-041663, R01 HD052732, and FP7-289346-EARLY NUTRITION.

\section{Financial Disclosures}

Elizabeth H. Blackburn, Jue Lin, and Elissa S. Epel are cofounders of Telomere Health, Inc., a company focused on telomere measurement. Assays and all other activity for the current report are, however, unrelated to this company. All other co-authors have nothing to declare.

\section{Disclosure Statement}

The authors have no conflict of interest relevant to this article to disclose.

\section{References}

1 Armanios M, Blackburn EH: The telomere syndromes. Nat Rev Genet 2012;13:693-704.

2 Zhu H, Belcher M, van der Harst P: Healthy aging and disease: role for telomere biology? Clin Sci (Lond) 2011;120:427-440.

3 Cawthon RM, Smith KR, O'Brien E, Sivatchenko A, Kerber RA: Association between telomere length in blood and mortality in people aged 60 years or older. Lancet 2003;361:393-395.

4 Aviv A: Genetics of leukocyte telomere length and its role in atherosclerosis. Mutat Res 2012;730:68-74.

5 Entringer S, Buss C, Wadhwa PD: Prenatal stress, telomere biology, and fetal programming of health and disease risk. Sci Signal 2012;5:pt12.

6 Heidinger BJ, Blount JD, Boner W, Griffiths K, Metcalfe NB, Monaghan P: Telomere length in early life predicts lifespan. Proc Natl Acad Sci U S A 2012;109:1743-1748.

7 Bateson M, Brilot BO, Gillespie R, Monaghan P, Nettle D: Developmental telomere attrition predicts impulsive decision-making in adult starlings. Proc Biol Sci 2015;282:20142140.

8 Asghar M, Hasselquist D, Hansson B, Zehtindjiev P, Westerdahl H, Bensch S: Chronic infection. Hidden costs of infection: chronic malaria accelerates telomere degradation and senescence in wild birds. Science 2015;347:436-438.

9 Daniali L, Benetos A, Susser E, Kark JD, Labat C, Kimura M, et al: Telomeres shorten at equivalent rates in somatic tissues of adults. Nat Commun 2013;4:1597.

10 Prescott J, Kraft P, Chasman DI, Savage SA, Mirabello L, Berndt SI, et al: Genome-wide association study of relative telomere length. PLoS One 2011;6:e19635.

11 Moores CJ, Fenech M, O'Callaghan NJ: Telomere dynamics: the influence of folate and DNA methylation. Ann N Y Acad Sci 2011; 1229:76-88.

12 Blasco MA: The epigenetic regulation of mammalian telomeres. Nat Rev Genet 2007;8: 299-309.
13 Richards JB, Valdes AM, Gardner JP, Kato BS, Siva A, Kimura M, et al: Homocysteine levels and leukocyte telomere length. Atherosclerosis 2008;200:271-277.

14 Paul L, Cattaneo M, D'Angelo A, Sampietro F, Fermo I, Razzari C, et al: Telomere length in peripheral blood mononuclear cells is associated with folate status in men. J Nutr 2009;139:1273-1278.

15 Antony AC: In utero physiology: role of folic acid in nutrient delivery and fetal development. Am J Clin Nutr 2007;85:598S-603S.

16 Economides DL, Ferguson J, Mackenzie IZ, Darley J, Ware II, Holmes-Siedle M: Folate and vitamin B12 concentrations in maternal and fetal blood, and amniotic fluid in second trimester pregnancies complicated by neural tube defects. Br J Obstet Gynaecol 1992;99: 23-25.

17 Lillycrop KA, Phillips ES, Jackson AA, Hanson MA, Burdge GC: Dietary protein restriction of pregnant rats induces and folic acid supplementation prevents epigenetic modification of hepatic gene expression in the offspring. J Nutr 2005;135:1382-1386.

18 Mikkola HK, Orkin SH: The journey of developing hematopoietic stem cells. Development 2006; 133:3733-3744.

19 UK NEQAS: Haematinic assays scheme. Annual report 2001. Sutton Coldfield, UK, NEQAS, 2002.

20 Pfeiffer CM, Fazili Z, McCoy L, Zhang M, Gunter EW: Determination of folate vitamers in human serum by stable-isotope-dilution tandem mass spectrometry and comparison with radioassay and microbiologic assay. Clin Chem 2004;50:423-432.

21 Simhan HN, Himes KP, Venkataramanan R, Bodnar LM: Maternal serum folate species in early pregnancy and lower genital tract inflammatory milieu. Am J Obstet Gynecol 2011;205:61.e1-e7.

22 Okuda K, Bardeguez A, Gardner JP, Rodriguez $\mathrm{P}$, Ganesh V, Kimura M, et al: Telomere length in the newborn. Pediatr Res 2002;52: 377-381.

23 Kimura M, Gazitt Y, Cao X, Zhao X, Lansdorp PM, Aviv A: Synchrony of telomere length among hematopoietic cells. Exp Hematol 2010;38:854-859.

24 Lin J, Epel E, Cheon J, Kroenke C, Sinclair E, Bigos M, et al: Analyses and comparisons of telomerase activity and telomere length in human $\mathrm{T}$ and $\mathrm{B}$ cells: insights for epidemiology of telomere maintenance. J Immunol Methods 2010;352:71-80.

25 Aviv A, Hunt SC, Lin J, Cao X, Kimura M, Blackburn E: Impartial comparative analysis of measurement of leukocyte telomere length/ DNA content by Southern blots and qPCR. Nucleic Acids Res 2011;39:e134.

26 Demissie S, Levy D, Benjamin EJ, Cupples LA, Gardner JP, Herbert A, et al: Insulin resistance, oxidative stress, hypertension, and leukocyte telomere length in men from the Framingham heart study. Aging Cell 2006;5: 325-330.

27 Valdes AM, Andrew T, Gardner JP, Kimura M, Oelsner E, Cherkas LF, et al: Obesity, cigarette smoking, and telomere length in women. Lancet 2005;366:662-664.

28 Paul L: Diet, nutrition and telomere length. J Nutr Biochem 2011;22:895-901.

29 Eisenberg DT, Hayes MG, Kuzawa CW: Delayed paternal age of reproduction in humans is associated with longer telomeres across two generations of descendants. Proc Natl Acad Sci U S A 2012;109:10251-10256.

30 Broer L, Codd V, Nyholt DR, Deelen J, Mangino M, Willemsen G, et al: Meta-analysis of telomere length in 19,713 subjects reveals high heritability, stronger maternal inheritance and a paternal age effect. Eur J Hum Genet 2013;21:1163-1168.

31 de la Rochebrochard E, Thonneau P: Paternal age and maternal age are risk factors for miscarriage; results of a multicentre European study. Hum Reprod 2002;17:1649-1656. 